\title{
Simple Household Space Design Based on Soft Decoration of Furniture
}

\author{
Zhang Nini ${ }^{1}$, Bu Leping ${ }^{1.2} *$, Meng Shaoqing ${ }^{1}$ \\ ${ }^{1}$ Guangzhou city Construction college, Guangzhou 510925, China \\ ${ }^{2}$ College of Mechanical and Electrical Engineering, Inner Mongolia Agricultural University, Hohhot 010018
}

\begin{abstract}
With the continuous development of China's economy, people's quality of life is constantly improving. As a matter of course, more and more attention is paid to home design. In particular, the space design of small apartments is welcomed by everyone. Among them, soft cloth decoration is a very popular form of home design now, which is very suitable for the design and use of small apartment. But the diversity of small family space brings difficulties to the design. The design and calculation of the simple home space in the small apartment with the RANSAC algorithm is fully satisfied with the requirements of the use of the algorithm and can achieve the accuracy of our calculation and facilitate communication with customers. The RANSAC algorithm has the powerful ability of image processing, which can help us to establish the visualization research of space design.
\end{abstract}

\section{Introduction}

With the continuous development of the national economy, the quality of life of the domestic people has been unprecedentedly raised. People have more and more requirements for the space design of home. People's aesthetic requirements and comfort require us to make a careful analysis of the design of home space (Dusmez Set al2017) ${ }^{[1]}$. In particular, the simple design of small apartment is hot now. Small apartment simple home furnishing design has many decoration styles, and the most popular one is cloth art soft clothes. This decoration style and its simple home design are applicable to small apartments. This kind of space design needs the aid of computer's image processing ability. It is difficult to visualize the space processing ability without computer, so this paper establishes a small household space design model based on RANSAC algorithm (Kaspi Oet al 2017) ${ }^{[2]}$. By using this kind of calculation model, it is easy to design simple home furnishing space based on soft furnishing.

In this paper, a brief analysis of the design of home furnishing space based on soft furnishing is first introduced. Then, the feasibility of using the RANSAC algorithm to calculate the household space design based on fabric soft decoration is studied (Kim H Het al2016) ${ }^{[3]}$. The practicability of the algorithm is proved through our analysis of feasibility. Then, the calculation form and the calculation step of the algorithm are studied. Based on the calculation formula of this paper, a simple home space design model based on the RANSAC algorithm is established (Liu Cet al2017) ${ }^{[4]}$. At the end of the paper, the algorithm's ability to calculate is tested, and the practicability and feasibility of the algorithm is proved by testing.

\section{State of the art}

Soft furnishing is widely used in small household design in the world and has been well received by everyone. This style of decoration is simple but not spacious, and is in line with people's aesthetic ideas nowadays (Frommholz Det al2016) [5]. In addition, RANSAC algorithm is a computer algorithm for image processing in the US in the 70 s of last century. This computer algorithm has an unprecedented ability to calculate and deal with images. In the face of image calculation, the image can be analyzed in the form of a matrix, which is accurate and efficient (Zhai Qet al2016) ${ }^{[6]}$. The computational research of RANSAC algorithm has been applied in practical engineering, which adds a new research path to the computation of image processing in the world (Sheng Het al2016) ${ }^{[7]}$

In the early 90s of last century, RANSAC algorithm was introduced into China (Syulistyo A Ret al2016) ${ }^{[8]}$. At that time, there was no excellent image processing algorithm in our country. The introduction of RANSAC algorithm fills the gap in the calculation of image processing in our country and quickly gets the recognition from all walks of life and develops vigorously. Since the beginning of this century, the use of RANSAC algorithm is more diversified. The use of medicine and other fields has brought new opportunities to the development of RANSAC algorithm (Shao T Tet al2016) ${ }^{[9]}$. At present, the calculation of the algorithm is also widely used in the 
construction industry. The application of decoration can increase the visualization of the decoration style. The calculation of home furnishing space for soft furnishing small apartments is extended from this point of view (Ayhan Bet al2017) ${ }^{[10]}$

\section{Methodology}

\subsection{A brief introduction to image stitching}

Small apartment decoration research needs the help of image mosaic technology. This kind of image stitching technology can make the whole room become a whole to facilitate our design and observation, which helps the design of the whole room, not the separate design of the room and can also improve the overall effect of the decoration. Below, the image mosaic technology used in this paper is analyzed and studied.

The image mosaic technology based on RANSAC algorithm used in this paper is a technology that is not affected by the angle of the pictures. This technology is very widely applied, and has great ability to process images such as charts, maps and cloud images. The overall image stitching technology contains many research directions and is very wide, so in this paper, it is the first task to study the image mosaics by choosing one direction and selecting a suitable research direction for the use of this paper. The analysis steps of image mosaic technology mainly include six modules. The six modules are analyzed by using Figure 1 below.

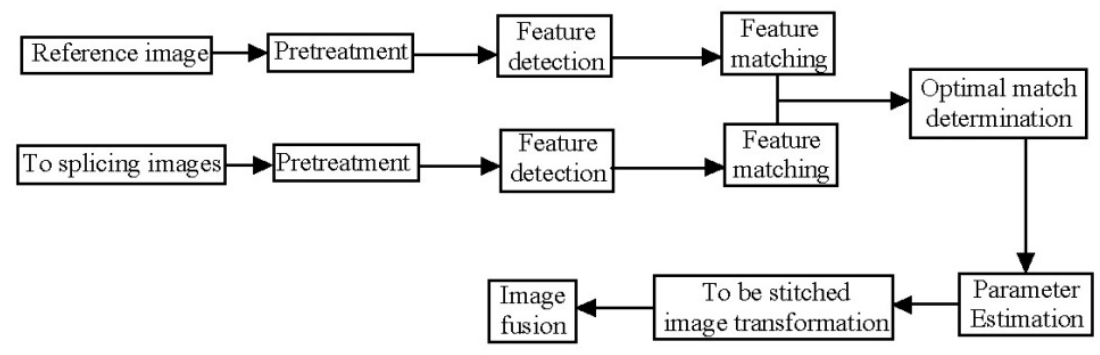

Figure.1 Analysis step of image mosaic technology

Image stitching needs to be done in a certain way. Otherwise, the images will be cluttered, which will affect our later processing. The first step is the extraction of feature points for image stitching. The images we have now studied are all made up of pixels, each of which has different pixel information, and all these information together make a whole image. The meaning of the feature point is that the pixels in the pixels have a representative pixel point, and the pixels are extracted and analyzed, and the later calculation operation can be carried out. But before extracting feature points, image pre-processing is the first step. Image pre-processing is mainly for two operations. The first is to eliminate interference from pixels. In the process of image scanning, there may be some mistakes and mistakes. In the image pre-processing stage, the noise information in the image needs to be eliminated. The advantage of this method is that we can make our later calculation fast, simplify the calculation difficulty and improve the accuracy of calculation. Image preprocessing can use different processing methods, but image enhancement is one of the most commonly used methods in our work. There are many key pixels and key information in the image. The role of image enhancement is to enlarge the role of these important pixels, and even increase the resolution of the image. The enhancement of the feature information in the image can help us grasp the key points and increase the computational efficiency in the later calculation. There are many ways of image enhancement, such as transform domain enhancement and wavelet transform enhancement. In this paper, the transform domain enhancement method is used.

Transform domain image enhancement method is to transform the image to the frequency domain, or to transform the image into the wavelet domain. In the frequency domain, the convolution theory is mainly used. The main ideas are:

$$
g(x, y)=s(x, y) \bullet h(x, y)
$$

Among them, $\mathrm{s}(\mathrm{x}, \mathrm{y})$ is the initial expression function of image and $\mathrm{h}(\mathrm{x}, \mathrm{y})$ is linear shift invariant operator. $\mathrm{g}(\mathrm{x}, \mathrm{y})$ is convolution of $\mathrm{s}(\mathrm{x}, \mathrm{y})$ and $\mathrm{h}(\mathrm{x}, \mathrm{y})$. After being denounce the Fourier transform, it can be gotten that:

$$
G(u, v)=H(u, v) \times S(u, v)
$$

Among them, $\mathrm{H}(\mathrm{u}, \mathrm{v})$ is the Fourier transformed function corresponding to $\mathrm{h}(\mathrm{x}, \mathrm{y})$, and $\mathrm{H}(\mathrm{u}, \mathrm{v})$ is the transfer function; $\mathrm{G}(\mathrm{u}, \mathrm{v})$ is the Fourier transform corresponding to $\mathrm{g}(\mathrm{x}, \mathrm{y}) ; \mathrm{S}(\mathrm{u}, \mathrm{v})$ is the Fourier transform corresponding to $\mathrm{s}(\mathrm{x}, \mathrm{y})$. And, $\mathrm{s}(\mathrm{x}, \mathrm{y})$ is the original image function, that is, $s(x, y)$ is a fixed value. In image enhancement, the transfer function $\mathrm{H}(\mathrm{u}, \mathrm{v})$ is required, and then $\mathrm{G}(\mathrm{u}, \mathrm{v})$ can be obtained through formula 2 . Then through the inverse operation, $g(x, y)$ is obtained. Concretely, it can be expressed by formula 3 .

$$
g(x, y)=S^{-1}[H(u, v) S(u, v)]
$$

Through our study of other image enhancement techniques, it is found that the computation of the transform domain enhancement method used in this paper can reduce the calculation pressure in the follow-up, and can show more powerful computing power in this paper. This is due to the characteristics of different images. In the image processing of the small household space design which needs to be calculated in this paper, the transform domain enhancement method is undoubtedly an image 
enhancement method that takes into account the accuracy of calculation and the efficiency of calculation.

\subsection{Simple household space design model based on RANSAC algorithm for soft decoration of cloth art}

In the previous paper, the pre-processing method of the RANSAC algorithm and the calculation steps are analyzed, and an image pre-processing method suitable for this paper is selected. Next, the analysis and research of the basic calculation process of RANSAC algorithm used in this paper need to be carried out.

The main form of the traditional RANSAC algorithm is based on a coordinate system. The whole image is in a coordinate system, which may be a rectangular coordinate system or other coordinate system and is determined according to the calculation. In the coordinate system, all data have their own coordinate points in the coordinate system. The analysis of characteristic points is also based on this coordinate system. When the traditional RANSAC algorithm is all the iteration number $\mathrm{N}$ of the inner point set, the calculation formula 4 is required under the premise of the confidence probability $\mathrm{P}=0.99$.

$$
N=\frac{\log (1-p)}{\log \left(1-(1-\omega)^{2}\right)}
$$

When solving transformation matrix, whether a specific point pair is the internal point we need first needs to be judged. The specific methods of judgment are as follows:

First, 4 the corresponding matching feature points on matching image 1 and image 2 are selected randomly. Then, according to the transformation matrix, the distance between the matching points in image 1 and image 2 is calculated, and the distance from Vanward P'to coordinate transformation after each transformation is transformed. The sum of two distances is used as the basis for judging the internal points. The sum of the distance is expressed by formula 5 .

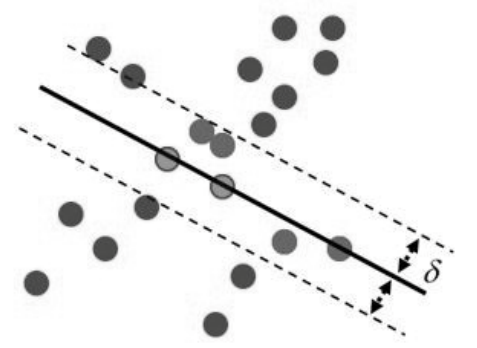

$$
|\boldsymbol{O}|=6
$$

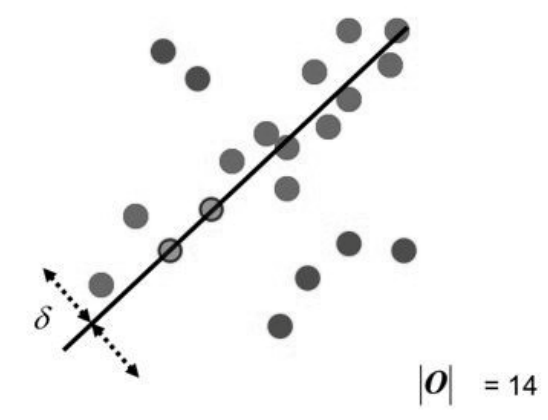

Figure.2 Selection pattern of feature points

The calculation process of the RANSAC algorithm in the above is a form of computation after the optimization of the algorithm according to the calculation requirements of this paper. This optimized RANSAC algorithm will be more suitable for the calculation requirements of this paper. But the algorithm is calculated and analyzed by

$$
d=\sum_{i}\left(d\left(\vec{p}, H \overrightarrow{p_{i}}\right)^{2}+d\left(\overrightarrow{p^{\prime}}, H \overrightarrow{p_{i}^{\prime}}\right)^{2}\right)
$$

The distance between the feature points is represented by formula 6 .

$$
D=\sqrt{\sum_{k=1}^{n}\left(D_{I k}-D_{I j}\right)^{2}}
$$

Reference value $\mathrm{T}$ is set. When the sum of distances between the corresponding two matching points in Fig. 1 and Fig. 2 is $<\mathrm{T}$, it is considered that the matching point is one of the interior points. Otherwise, the match point is abandoned. The steps of the specific method are as follows:

(1) 4 pairs of feature points are randomly extracted, and the parameter model matrix Hcur is calculated based on the extracted feature points.

(2) According to the parameter model matrix, formula 5 is used to calculate the distance between the matching points in image 1 and image 2. When the distance test is less than $\mathrm{T}$, it is determined as the interior point, and the number of statistics points is $\mathrm{S}$.

(3) If $\mathrm{S}$ is greater than $\mathrm{M}$ inlier, it is considered that the current parameter model matrix is the best matrix $\mathrm{H}=$ Hcur. And the inner point is preserved; The $\mathrm{M}_{-}$inlier here is the number of points calculated in the previous time.

(4) Formula 4 is used to calculate the number of iterations $\mathrm{N}$, and continuous cycle 1 to 3 . When the set of internal points reaches the maximum and no longer changes, the loop stops. The parameter matrix $\mathrm{H}$ is calculated by the maximum interior point set. The matrix obtained is the most accurate parameter model matrix. In order to show the selection of feature points more clearly, the final feature selection pattern is shown in Figure 2 below. computer, so a computer calculation model can be established through the analysis of the calculation formula.

Through the calculation of the computer computing model based on the RANSAC algorithm, it will be more convenient and accurate for the study of the simple home space design for the small type of the cloth. Through this 
calculation form, the complete home space design can be carried out and the design speed is increased, and it can better communicate with customers.

\section{Result analysis and discussion}

According to the requirements of this paper, a new form of RANSAC algorithm is established, and the computation model based on this RANSAC algorithm is established. In fact, our ability to compute the algorithm has not yet been determined. In order to extend this computing model and increase its market competitiveness, the RANSAC algorithm needs to carry out the test experiment. Through the test experiments in this paper, it is proved that the algorithm is really applicable to the calculation of simple household space design in soft furnishing, and the test begins below. The test environment diagram is shown in Figure 3 below.

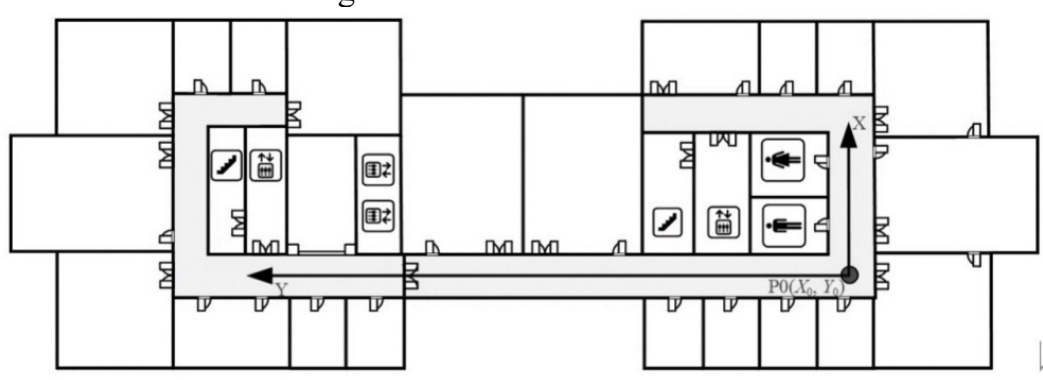

Figure. 3 test environment

First, the test in this paper is the ability to extract the key points when the algorithm is used to calculate. For this test, five algorithms are used for unified testing and four control groups are used as an experimental group. The results of the experiment are shown in Table 1 below.

Table.1 Results of experimental data in this article

\begin{tabular}{|c|c|c|c|c|c|c|}
\hline Extraction Method & a & $\mathrm{b}$ & $\mathrm{c}$ & $\mathrm{d}$ & $\begin{array}{l}\text { Plane } \\
\text { points/noise } \\
\text { points }\end{array}$ & $\begin{array}{l}\text { Extraction } \\
\text { points }\end{array}$ \\
\hline Setting parameters & 0.57 & 0.57 & 0.57 & -0.57 & $900 / 100$ & 745 \\
\hline Least Squares & 0.58 & 0.60 & 0.54 & -0.58 & $900 / 100$ & 457 \\
\hline Moving least squares & 0.59 & 0.58 & 0.56 & -0.57 & $900 / 100$ & 687 \\
\hline Hough Transform & 0.57 & 0.59 & 0.55 & -0.56 & $900 / 100$ & 357 \\
\hline RANSAC & 0.58 & 0.57 & 0.58 & -0.57 & $900 / 100$ & 887 \\
\hline
\end{tabular}

In the above table, 900 computing points are calculated and 100 noise items are interfered. A total of 1000 key points were studied. Then the analysis of the last node extraction number shows that the calculation of the RANSAC algorithm used in this paper can extract 887 useful points. The accuracy of key point extraction is quite high. In contrast, the other four control groups had only 357 points in the Hough Transform with the least experimental results. Although the best least square algorithm has reached 745 , the computation time is much lower than the RANSAC algorithm used in this paper. It can be seen that the RANSAC algorithm used in this paper can accurately extract pixel points in a moderate computing environment. However, the extraction and computation of this pixel is only the first step in the algorithm calculation, and also the simplest step in computation. The later algorithm needs to be tested. The emphasis of the test is computation time and computation accuracy. Through the test above, it can be found that the two algorithms with the highest accuracy of pixel selection is the least square algorithm and the RANSAC algorithm used in this paper. The following tests are also aimed at comparing the two algorithms.

The first step is to test the computation time, which is related to the computational efficiency. It is enough to only study the computation time of the whole calculation. The test is divided into five experimental groups. The experimental variables of the five experimental groups are calculated. The calculation time test comparison diagram is shown in Figure 4 below. 


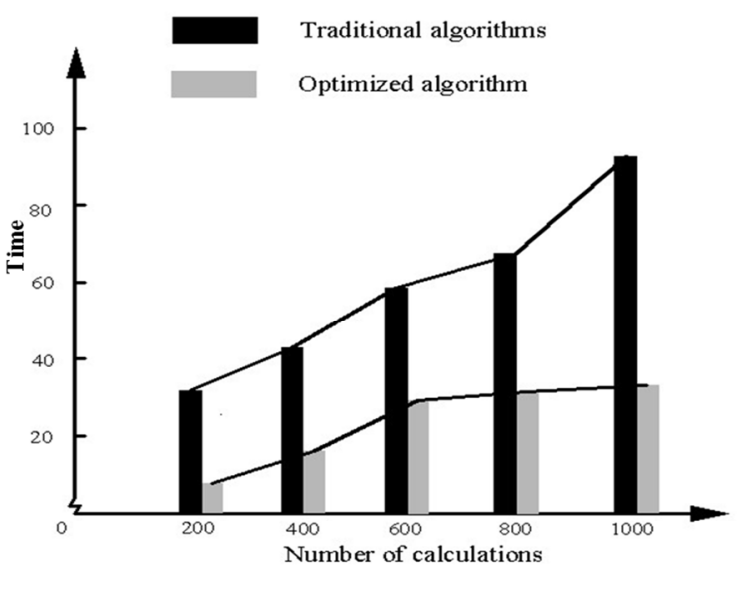

Figure. 4 Comparison of calculation time test

It can be seen clearly from the test results in the above picture the computation time of the RANSAC algorithm used in this paper is much less than the computation time of the least square algorithm. The computation time increased from only 7 seconds to 34 seconds. Compared with the computation time of the least square algorithm, the computational efficiency increases by three times. Moreover, the computation time increase rate of RANSAC algorithm used in this paper is also relatively small, which can be analyzed by calculating the rate of change between times.

In addition, in order to make the test of the algorithm more convincing, the accuracy of the algorithm is tested. For testing the accuracy of computation, the test environment for testing the computation time will continue to be used. Moreover, the accuracy of calculation points is analyzed separately, and the results are analyzed by using Figure 5 below.

It can be seen that the calculation accuracy of the RANSAC algorithm used in this paper does not change obviously with the increase of the calculation item. Although it has fallen, it has been in $90 \%$.But from the calculation of least squares algorithm controls the accuracy of the test and analysis, it can be seen that the algorithms accuracy decrease very badly. In the last group of tests, the accuracy of the least square algorithm has dropped to less than $60 \%$. Through the test above, it can be found that the RANSAC algorithm used in this paper is fully consistent with the calculation requirements of this paper and the calculation results are good.

\section{Conclusion}

With the continuous improvement of people's quality of life, people's aesthetic requirements for housing have gradually increased. Small apartment now occupies a large proportion of the room. The design research on small apartment simple home space has gradually entered our field of vision. Among them, the soft decoration of cloth art is very popular. So in this paper, a simple design model of home furnishing space based on RANSAC algorithm is established. Through the test of RANSAC algorithm, it is found that the correct rate of correct pixel selection is very high. By testing the accuracy and time of our algorithm, it is proved that the result of RANSAC algorithm is feasible.

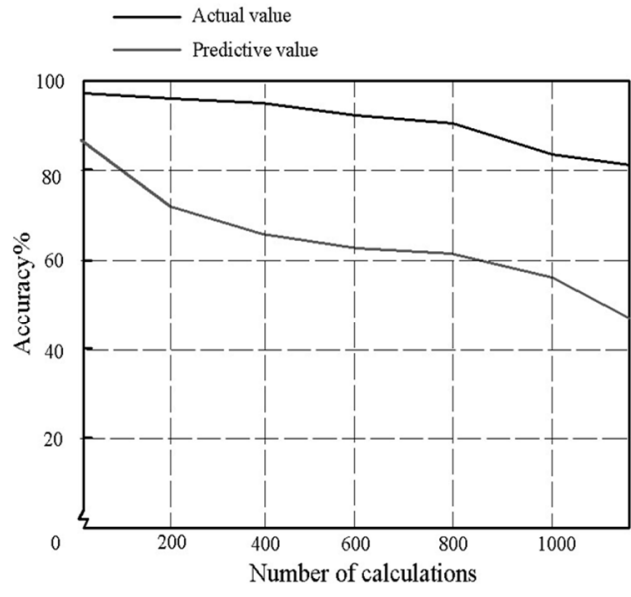

Figure.5 Calculation accuracy test comparison char

The accuracy of calculation has been stable over $90 \%$, and the computational efficiency is much better than that of the control group. In general, the design and calculation of the simple home space in the small apartment with the RANSAC algorithm is fully satisfied with the requirements of the use of the algorithm and can achieve the accuracy of our calculation and facilitate communication with customers.

\section{References}

1. Dusmez S, Heydarzadeh M, Nourani M, et al. Remaining Useful Lifetime Estimation for Power MOSFETs Under Thermal Stress With RANSAC Outlier Removal[J]. IEEE Transactions on Industrial Informatics, 2017, PP(99):1-1.

2. Kaspi O, Yosipof A, Senderowitz H. RANdom SAmple Consensus (RANSAC) algorithm for material-informatics: application to photovoltaic solar cells:[J]. Journal of Cheminformatics, 2017, 9(1):34.

3. Kim H H, Ko B C, Nam J Y. Predicting chlorophyllusing Landsat 8 OLI sensor data and the non-linear RANSAC method - a case study of Nakdong River, South Korea[J]. International Journal of Remote Sensing, 2016, 37(14):3255-3271.

4. Liu C, Shen Q, Pan H, et al. Modelling and simulation: an improved RANSAC algorithm based on the relative angle information of samples [J]. International Journal of Modelling Identification \& Control, 2017, 28(2):144.

5. Frommholz D, Linkiewicz M, Poznanska A M. Inlining 3d Reconstruction, Multi-Source Texture Mapping and Semantic Analysis Using Oblique Aerial Imagery [J]. ISPRS - International Archives of the Photogrammetry, Remote Sensing and Spatial Information Sciences, 2016, XLI-B3:605-612.

6. Zhai Q, Hu J, Yan X, et al. Modified RANSAC method for Three-dimensional scattering center extraction at a single elevation[J]. Progress in Electromagnetics Research B, 2016, 68(1):89-103.

7. Sheng H, Gao Y, Zhu B, et al. Feature extraction of SAR scattering centers using M-RANSAC and 
STFRFT-based algorithm[J]. Eurasip Journal on Advances in Signal Processing, 2016, 2016(1):46.

8. Syulistyo A R, Aprinaldi, Bowolaksono A, et al. ELLIPSE DETECTION ON EMBRYO IMAGING USING RANDOM SAMPLE CONSENSUS (RANSAC) METHOD BASED ON ARC SEGMENT[J]. International Journal on Smart Sensing \& Intelligent Systems, 2016, 9(3).

9. Shao T T, Kitamura M, Nakayama Y, et al. Random sample consensus combined with partial least squares regression (RANSAC-PLS) for microbial metabolomics data mining and phenotype improvement[J]. Journal of Bioscience \& Bioengineering, 2016, 122(2):168-175.

10. Ayhan B, Dao M, Kwan C, et al. A Novel Utilization of Image Registration Techniques to Process Mastcam Images in Mars Rover With Applications to Image Fusion, Pixel Clustering, and Anomaly Detection [J]. IEEE Journal of Selected Topics in Applied Earth Observations \& Remote Sensing, 2017, PP (99):1-12. 